\title{
A REPOSITORY OF INFORMATION VISUALIZATION TECHNIQUES TO SUPPORT THE DESIGN OF 3D VIRTUAL CITY MODELS
}

\author{
C. Métral ${ }^{\text {a }} *$, N. Ghoula ${ }^{a}$, V. Silva ${ }^{\text {a }}$, G. Falquet ${ }^{a}$ \\ ${ }^{\text {a }}$ Centre Universitaire d'Informatique, University of Geneva, 7 route de Drize, CH-1227 Carouge, Switzerland - \\ (claudine.metral, nizar.ghoula, vitor.silva, gilles.falquet)@unige.ch
}

KEY WORDS: 3D visualization, information visualization, 3D city model, 3D virtual environment, 3D GIS, knowledge base

\begin{abstract}
:
Virtual 3D city models are used for different applications such as urban planning, navigation, pedestrian behaviour, historical information, and disaster management. These applications require rich information models that associate urban objects not only with their geometric properties but also with other types of information. When designing such models the choice of visualization techniques is far from trivial because the city objects must be displayed together with additional information, such as historical facts, planning projects, pollutant concentration, noise level, etc. Finding relevant techniques depends on a set of criteria such as the type of information, but also on the tasks that will be performed and the associated context. Furthermore, a technique that is relevant when used in isolation may generate visual incompatibilities when used in conjunction with another one. We have defined a model for the representation of information visualization techniques in 3D city models. We have implemented this model in the form of an ontology and a knowledge base of techniques currently used in 3D city models or 3D GIS. The goal of such an approach is to provide a knowledge repository to support the design of $3 \mathrm{D}$ virtual city models in which non-geometric information must be presented. In this paper we describe the model and the ontology of information visualization techniques that we designed. We also show how the associated knowledge base can be used for the selection of visualization techniques depending on different criteria including task and context, and for the detection of visual incompatibilities between techniques when used in the same scene.
\end{abstract}

\section{INTRODUCTION}

\subsection{Motivation}

More and more applications based on 3D virtual city models are used for tasks that go far beyond the mere visualization of the city objects. These tasks generally require (1) rich information models that associate urban entities not only with their geometric properties but also with other types of information related to the city (pollutant concentration, road labels, historical facts, etc.) and (2) appropriate visualization techniques. Appropriate techniques rely on a set of criteria such as (1) the data type to display, (2) the tasks that will be performed by the users and (3) the associated context (level of detail, type of navigation, etc.). But, in the literature related to applications of 3D city models, the techniques used are too often not explicitly described. It is thus difficult to select a technique according to precise criteria or to compare techniques. Furthermore complex tasks can imply visualizing several types of data at once (e.g. air quality, noise, spatial distribution of pedestrian movement) but a technique that is efficient when used alone can induce visual incompatibilities when used in conjunction with others (visual occlusion, etc.). Those incompatibilities have to be taken in account when selecting visualization techniques.

Therefore, the selection of an effective and efficient visualization technique for a $3 \mathrm{D}$ environment is an important and difficult task. The aim of the work presented here is precisely to build a knowledge base of visualization techniques that can serve several purposes, in particular:

- to help a 3D virtual environment designer choosing the most appropriate visualization techniques for the intended users tasks and context (for instance, when designing a 3Denabled website to let citizen and professionals explore a new urban project)

- to help a 3D GIS user select a visualization technique to display a given dataset in the 3D model. The GIS system must be able to find and propose appropriate visualization techniques for each dataset, depending on the data types, and the context of use.

\subsection{Overview}

In this paper we address the problem of designing and implementing a usable knowledge base of information visualization techniques in $3 \mathrm{D}$ geographic environments, in particular in 3D city models. The main results we obtained are: (1) a model of information visualization techniques for 3D city models, and (2) an initial version of a knowledge base that implements this model, represents a number of existing techniques, and provides tools for finding techniques and computing incompatibilities.

The paper is organized as follows:

- Section 2 presents a brief state of the art on the modelling of visualization techniques. It covers the representation of the techniques themselves and the representation of their evaluation.

- Section 3 describes how we identified criteria that characterize information visualization techniques by studying a corpus of scientific articles related to visual applications of 3D city models. Then it presents a high level model for the description of the techniques and their evaluation.

\footnotetext{
* Corresponding author.
} 
- Section 4 presents the implementation of a knowledge base that represents visualization techniques and provides functions for (1) the selection of techniques depending on different criteria including task and context and (2) the detection of visual incompatibilities (and of their cause) between techniques used together. The knowledge base is in fact an OWL ontology that has been populated with 3D visualization techniques currently used in $3 \mathrm{D}$ city models or 3D GIS.

- In the final section we discuss the quality of the obtained knowledge base and propose future directions for its development and its use in existing applications

\section{BACKGROUND AND RELATED WORK}

This section summarizes previous work that study and classify visualization techniques. We also present important results on the evaluation of these techniques. In fact, selecting an appropriate technique depends not only on the intrinsic characteristics of the technique but also on their previous use and evaluation in different contexts. Since ontologies play an important role in the classification of visualization techniques and in the implementation of the proposed knowledge, this section begins with a brief presentation of this topic.

\subsection{Ontologies and knowledge bases}

Many definitions have been published for defining ontologies. in the context of computer and information sciences. Gruber (2009) defines an ontology as "a set of representational primitives with which to model a domain of knowledge or discourse. The representational primitives are typically classes (or sets), attributes (or properties), and relationships (or relations among class members)." Knowledge bases are obtained by feeding ontologies with knowledge about particular elements (usually called individuals), their attributes and their relationships to other individuals. Ontologies (and knowledge bases) differ from databases in the sense that "the languages of ontologies are closer in expressive power to first-order logic than languages used to model databases. For this reason, ontologies are said to be at the "semantic" level, whereas database schema are models of data at the "logical" or "physical" level." (Gruber, 2009). Moreover such a formalized ontology can be associated to a reasoner that can perform some computational reasoning.

In our case, an ontology-based approach enables (1) a formal representation of existing (but scattered) knowledge about 3D visualization techniques and (2) logical reasoning that allows some computation.

\subsection{Visualization ontologies or taxonomies}

Different classifications, terminologies, taxonomies or ontologies have been defined in the field of visualization, with different aims. Gao et al. (2008) presented the design of a visualization ontology, which aims at providing more semantics for the discovery of visualization services. The Top Level Visualization Ontology (TLVO) defined by Brodlie \& Noor (2007) aims at providing a common vocabulary to describe visualization data, processes and products. Based on an analysis of visualization taxonomies and on more recent work in visualization ontologies, Morell Pérez et al. (2011) propose some modifications to the TLVO in order to better represent the visualization process and data models. Voigt \& Polowinski (2011) aim at developing a unifying ontology applicable in visualization systems. Bazargan and Falquet (2009) have proposed to use description logics to represent the usability of techniques in a given context and to reason about it.

More recently, Voigt et al. (2012) have created a visualization ontology that supports a recommendation system for the selection of visualization components.

In our case, the ontology aims at organizing and formally representing visualization techniques for 3D city models. Existing ontologies cannot be reused because they mainly focus on the visualization process and not on the required items for an effective representation and reuse of visualization techniques.

\subsection{Evaluation representation}

Evaluating a visualization technique for some user tasks and for a specific context refers to the usability of the technique. From an exhaustive review of literature, Hornbæk (2006) defined usability aspects in terms of effectiveness, efficiency and satisfaction, the usability being the effectiveness, efficiency, and satisfaction with which specified users could achieve goals in particular environments. Bowmann et al. (2004) defined a classification of usability evaluation methods for virtual environments. He differentiated three criteria: (1) the user involvement (whether the evaluation requires or does not require users), (2) the context of evaluation (the context being either generic or application specific) and (3) the type of results (quantitative or qualitative). By combination, those criteria define height types of evaluation methods.

Several authors have defined a taxonomy of tasks in the field of information visualization. Amar et al. (2005) have defined lowlevel tasks that capture the activities of people trying to use information visualization tools for understanding data. Lee et al. (2006) have defined a taxonomy for viewing graphs. In this approach, complex tasks are considered as a series of low-level tasks.

In order to access information in $3 \mathrm{D}$ virtual environments, a user has to perform manipulation and locomotion. Tyndiuk (2005) defined six different types of spatial configuration from two reference frames: the user's viewpoint reference frame and the movement reference frame. According to the first one the user can be inside or outside the scene while the second one refers to the movement whether it derives from the camera, from the object or from the scene itself. Thereby, whether the user is inside or outside the scene, he/she can move, manipulate objects or manipulate the scene itself. For 3D urban models manipulation and/or locomotion is most often done according to a viewpoint close to the ground (snail view or pedestrian level) or by flying through or over the model (bird's eye view) (Vaaraniemi et al., 2013).

\section{A MODEL OF 3D INFORMATION VISUALIZATION TECHNIQUES}

\subsection{Knowledge sources}

We studied the scientific literature about applications based on $3 \mathrm{D}$ city models to obtain a global view of the domain. Although the techniques used in these applications are often not explicitly described, they provided enough information to draw initial classification axis. The studied models and applications were used for various tasks, such as:

- Evaluation of the wind comfort for pedestrians in a city street Amorim et al. (2012) where 3D coloured polylines (colour representing wind velocity) are added to the geometrical model. 
- Assessment of air quality in a street or neighbourhood by adding coloured solid objects to the 3D buildings Lu et al. (2009), San José et al. (2012).

- Estimation of vehicle traffic from Yatskiv and Savrasovs (2012) by adding 3D objects (vehicles) to "animate" the movement paths.

- Analysis of pedestrian behaviour Marina et al. (2012) where colored bars visualize spatial distribution of pedestrian movement.

- Analysis of human perception of space (Fisher-Gewirtzman, 2012) where colored lines represent visual exposure or visual openness in the 3D city model.

- Visualization of historically enriched 3D city models where information (text and images) has been added to the geometrical model Alamouri and Pecchioli (2010), Hervy et al. (2012).

- Emergency evacuation of buildings where a routing network is superposed to the building (Atila et al, 2013)

This study showed that visualization techniques could be classified along three main axes: the kind of data to be visualized (the input data); the visual rendering (how data are displayed in the $3 \mathrm{D}$ scene); and the usage of the technique, in terms of context and task.

\subsection{Data representation}

The data types are various, ranging from rich text, such as in a pdf file where images can also be present Hervy et al. (2012), to $3 \mathrm{D}$ scalar values (temperature) or to $3 \mathrm{D}$ vector values (wind) San José et al. (2012). An important point to note is that these data are spatialized. Each data element is associated to some spatial region. In a vector field each vector is associated to a $3 \mathrm{D}$ point, in an energy consumption dataset, each value (in $\mathrm{kWh}$ ) is associated to a building, in the output of a computational fluid dynamics model each value is the average fluid speed in a $3 \mathrm{D}$ cell, etc.

Moreover, all the coverage regions of an input dataset may be located within a specific spatial object, as is the case for a vector field on a surface, or measurement values at points forming a grid.

In our model, the DataDescription class represents the characteristics of the input data (see Figure 1). A data description has a data type, a coverage, and an information type. The representation of a data type is an object that belongs to the DataType subclass hierarchy (only partially shown on Figure 1). The type hierarchy is organized according to the programming language theoretic notion of subtype (a subtype can be used wherever a super-type is expected); it does not correspond to set inclusion. As we will see in the next section, this organization fits well with the intended query semantics on the knowledge base.

The coverage is the spatial region to which a data value is attached. It is either a geometric element (point, curve, etc.) or an urban object of the city model. The representation of a coverage is therefore an object in one of the subclass of InputLocation. This subclass hierarchy is the union of the geometric classes found in the GML Geography Markup Language (OGC 07-036, 2007) and the city objects found in CityGML (OGC 12-019, 2012).

The containedIn association may be used to specify that the coverage elements are all contained in a (not necessarily connected) spatial object.

The same data types (e.g. a scalar field) may represent completely different information types (e.g. measured temperatures or pollutant concentration in an urban area). Since different information types are generally used in different contexts and for different tasks, it is not obvious that a technique suitable for an information type will also be appropriate for another one (even though they are represented with the same data type). Therefore it is important to describe not only the data type but also the information type for which the technique has been primarily designed. Since the notion of information type is related to some knowledge of the real world, each information type object is related to WordNet concepts (WordNet is a lexical ontology and contains all the senses of the English words) Miller (1995).

Figure 2 shows the data description for a technique intended to display a set of real numbers that represent pollutant concentrations in 3D regions arranged in a grid (see Figure 4 for an illustration of this technique).

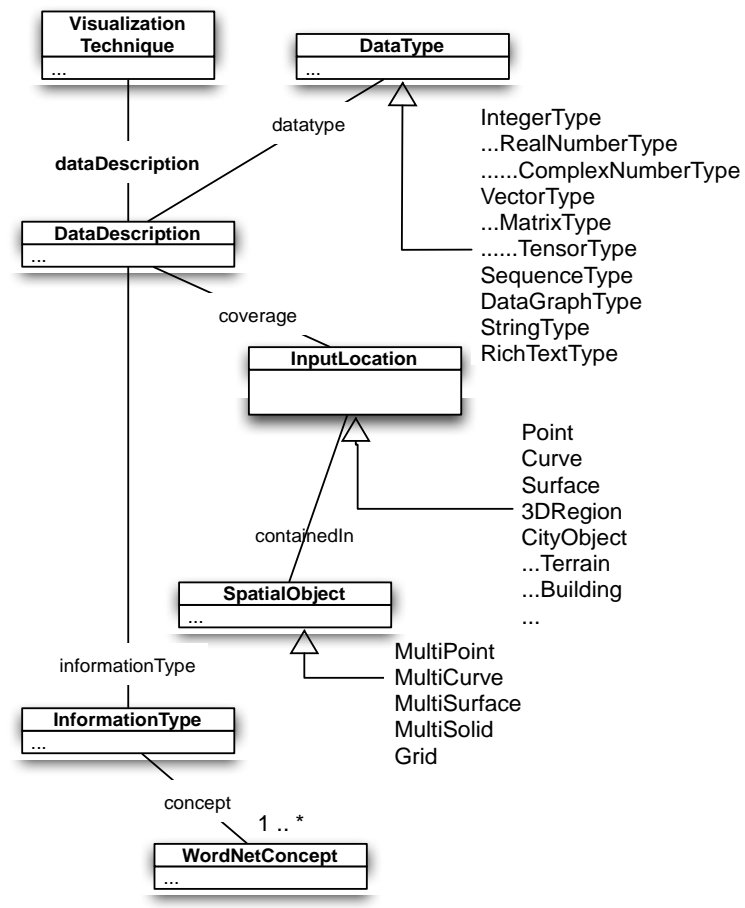

Figure 1. DataDescription representation

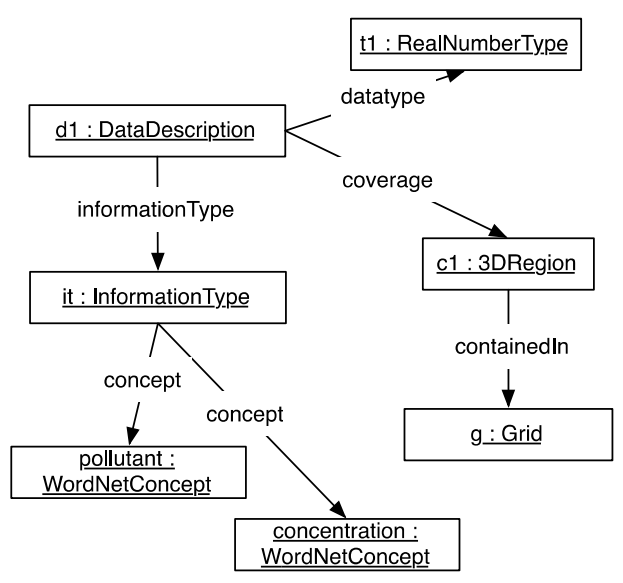

Figure 2. Description of the input data of a technique

\subsection{Output rendering representation}

The information to visualize is not directly associated to a geometry that could be used to visualize them. Each input data has thus to be associated to a visual rendering (output shape) 
that will be placed at a particular output location. The output location describes whether the output rendering takes place directly in the 3D space (3DSpace) or whether it is attached to an object of the model (ObjectAttached) where the objects are those described in CityGML (OGC 12-019, 2012). In this last case a spatial relation can be specified (above, below, etc.)

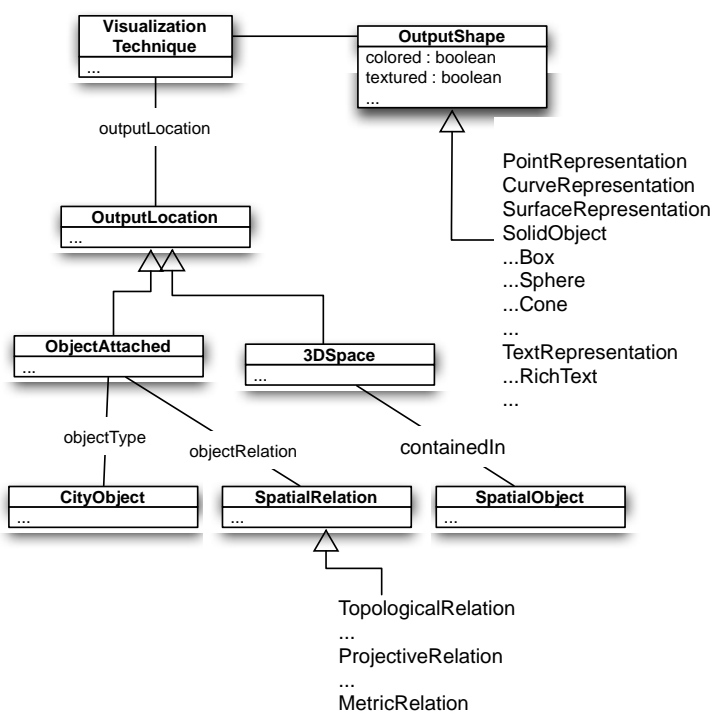

Figure 3. OutputRendering representation

Figure 5 shows the description of air quality visualization as colored spheres positioned on a surface ( $1 \mathrm{~m}$ above terrain level) while Figure 4 shows an illustration of the described technique.

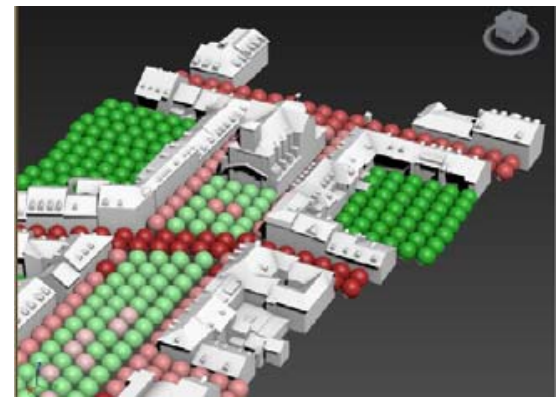

Figure 4. An air quality visualization technique

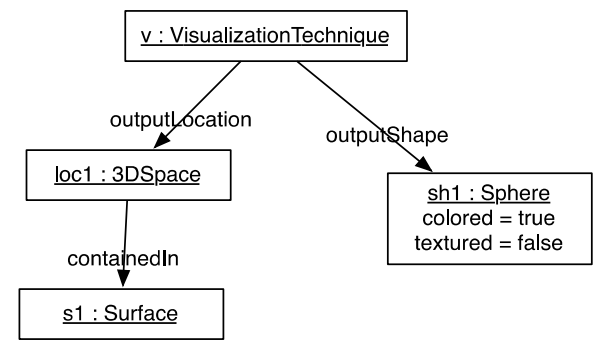

Figure 5. Description of the output rendering of an air quality visualization technique

\subsection{Usage representation}

In our case, it is better to talk of usage instead of usability since many techniques are widely used without any formal evaluation.

As previously seen (see section 2.3), the tasks can be organized in high-level tasks composed of low-level tasks. The urban tasks performed by the users can be considered as high-level tasks. Performing them implies navigation tasks or access-todata tasks in the 3D model (visual tasks). Thus, the user task Analysis of pedestrian behaviour implies retrieving the highest (or lowest values) of a representation of integer type. In order to determine precisely the access-to-data tasks, we started from the work performed by Lee et al. (2006). We then adapted the tasks defined by the authors (1) according to the data type (text, numeric data) and (2) according to what is really expected as result. Indeed, we may want to determine the exact value of the data that is presented, or determine approximately its value (by placing it in a range of value), or determine which values are the largest or the smallest ones. Thus we have defined the following tasks: ReadValue, ReadText, Estimate, Compare, etc. The contexts most commonly encountered in the 3D urban models studied are usually not defined in a formal and precise way. In order to define such contexts we started from previous work (see section 2.3). We thus have the following elements: a spatial configuration and a viewpoint (Bird's eye view, Pedestrian level). We then added two more elements: a navigation space and a city model. The navigation can be free or restricted to a navigation space (SpatialObject). The CityModel class describes the characteristics of the 3D city model (in terms of level of details, geographic name, location, etc.) We also have an attribute describing the display type. Indeed, the outcome of the evaluation of a technique may significantly differ, depending on the context.

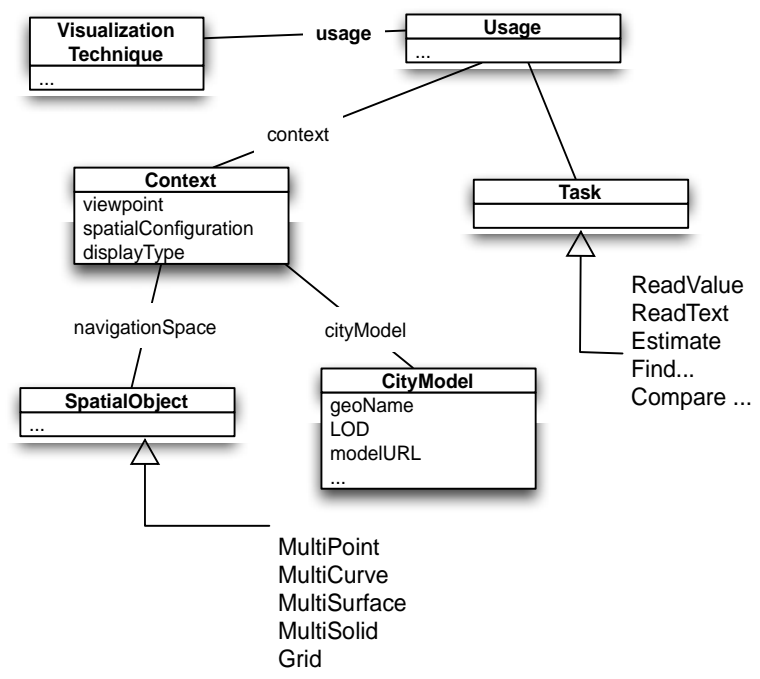

Figure 6. Usage representation

Figure 7 shows a technique that has been defined for reading texts when navigating at pedestrian level along curves.

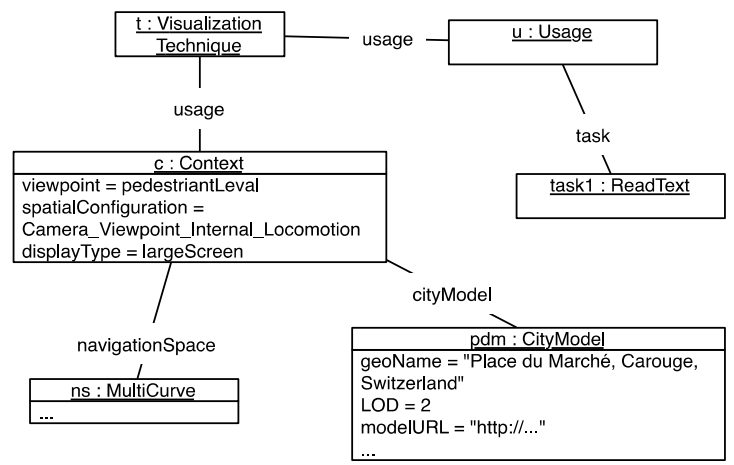

Figure 7. Description of the usage of a technique 


\section{IMPLEMENTATION OF THE KNOWLEDGE BASE}

This section presents the implementation principles of a usable knowledge base that is based on the model presented in the previous section. The knowledge base must satisfy at least two important requirements:

1. to have an expressive representation language that makes use of the vocabulary defined in the conceptual model and that is sufficient to express complex technique descriptions, such as "techniques with an output location that is a Street or a Building but not a WaterBody"

2. to express and solve queries by using logical inference. For instance, when looking for a technique that uses solid objects as output shapes, a technique that uses cones or spheres should be selected (because cones and spheres are solid objects)

For these reasons, and for others mentioned below, it is highly suitable to use a formal ontological language, such as OWL (OWL Working Group, 2012), and an ontology management system (including an OWL reasoner) to implement the knowledge base.

\subsection{An ontology-based implementation}

The first part of the implementation consists in translating the UML conceptual schema into OWL definitions. For the proposed model this can be done in a very straightforward way by applying the following translation scheme (some UML constructs may be hard to represent in OWL, but they don't appear in this model):

- UML class or association class $\rightarrow$ OWL class

- UML simple association $\rightarrow$ OWL object property (+ some, only, min, or max constructs to represent number constraints)

- UML attribute (integer, string, boolean valued) $\rightarrow$ OWL datatype property

- UML subclasses $\rightarrow$ OWL subClassOf axioms

This translation provides the basic vocabulary that will be used to represent the visualization techniques.

An interesting feature of the OWL language is its ability to define new classes by combining already defined classes through logical operators. Therefore, the description element mentioned in point 1 here above (techniques with an output location that is a Street or a Building but not a WaterBody) can be represented as:

\section{VisualizationTechnique \\ and (outputLocation some (ObjectAttached and objectType some \\ (Street or Building) and (not WaterBody)))}

\subsection{Formal representation of $3 D$ information visualization techniques}

A technique is represented as an instance of the VisualizationTechnique class, linked to instances of the DataDescription, OutputLocation and OutputShape classes that describe its characteristics.

The following expression describes a technique, illustrated on Figure 8, that presents texts relative to buildings as text panels located on a street.

\author{
VisualizationTechnique \\ and (outputlocation some (ObjectAttached \\ and objectType some Street)) \\ and (outputshape some RichTextRepresentation)
}
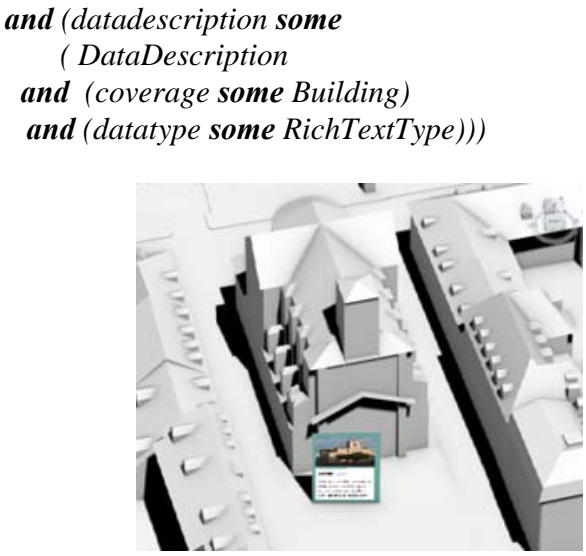

Figure 8. A technique for the visualization of rich text panels

Similarly, (Figure 9) shows a technique that uses cones of different heights positioned on streets or squares to represent the number of pedestrians passing at different locations. Its formalized description can be:

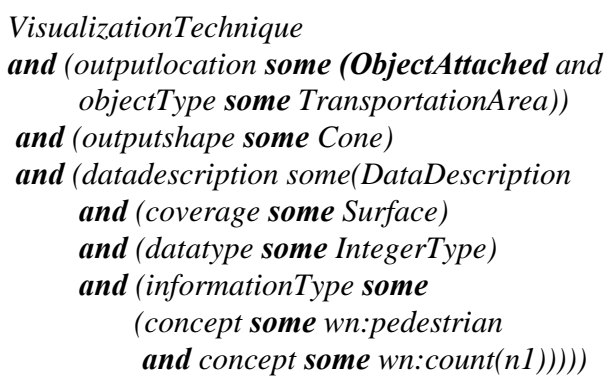

Here wn:pedestrian and wn:count(n1) are classes imported from the WordNet ontology. Since the word count has several senses, the ( $n 1)$ suffix indicates that we refer to the first meaning of count as a noun (the total number counted).

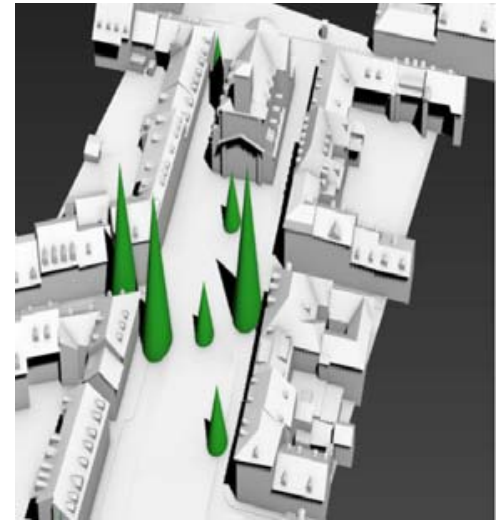

Figure 9. A technique for the visualization of pedestrian numbers

\subsection{Querying the knowledge base}

The main query task consists in finding techniques that are suitable for a particular dataset and context of use. This amounts to describe the characteristics of the desired technique in the form of a class specification and to let the logical reasoner infer which techniques belong to this class. 
The following expression is intended to find techniques to represent integer values that are located at specific points in a grid.

\author{
VisualizationTechnique \\ and dataDescription some (DataDescription \\ and datatype some IntegerType \\ and coverage some Point \\ and containedIn some Grid)
}

Since RealNumberType has been defined as a subclass of IntegerType (see 3.2), this query will also return techniques that can represent real numbers. This is what we want since a technique that can display real numbers will certainly work for integer numbers. And this is precisely what an OWL reasoner will do.

Similarly, this query will also return techniques that have more specific coverage or containedIn properties. For instance, a technique to display numbers that are located at points in an orthogonal grid (a special type of grid)

The following example query finds techniques for the estimation of air pollutant concentration with a navigation context above the scene or in the scene (at pedestrian level)

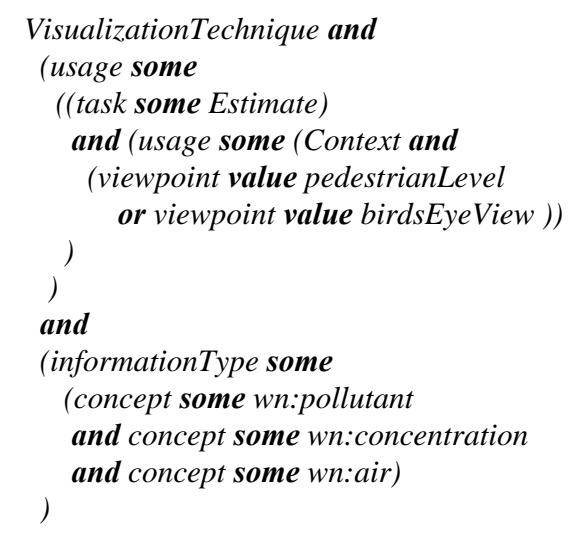

In this case, the system will be able to retrieve, for instance, techniques that were used to estimate the concentration of nitrogen oxide or sulfur dioxide in the air (since these are subclasses of Pollutant).

This type of query cannot be expressed in standard relational or $\mathrm{xml}$ databases because they do not take into account the notion of subclass and the related inference rules.

\subsection{Detection of incompatibilities between techniques}

The term incompatibility defines the impossibility to use several visualization techniques together. For instance, a texture rendering technique cannot a priori be used with another technique with the same type of rendering because there is a risk of occlusion and thus of confusion when interpreting the information in case they are attached to the same city object. However a texture rendering technique can be potentially used with a technique having a different type of rendering (display panel for example).

The detection of incompatibilities between techniques is difficult or even impossible to express in the OWL language. However, it is possible to write inference rules in the SWRL rule language ${ }^{1}$ to generate incompatibility relations.

\footnotetext{
${ }^{1}$ SWRL is a combination of OWL and RuleML expressing
} rules on ontological entities (Horrocks et al., 2004).
For instance, if the output location of two techniques $t 1$ and $t 2$ is above objects of the same type and their output shape are the same (e.g. they both use boxes above buildings) then they are incompatible. This rule can be written as follows in (simplified) SWRL:

$$
\begin{aligned}
& \text { outputLocation(t1, ol1) and outputLocation }(t 2, \text { ol2) } \\
& \text { and objectType }(o 1, \text { ot1) and objectType }(o 2, \text { ot } 2) \\
& \text { and ot } 1=\text { ot } 2 \text { and objectRelation }(o 1, r 1) \\
& \text { and objectRelation }(o 2, r 2) \text { and } r 1=r 2 \\
& \text { and outputShape }(t 1, s 1) \text { and outputShape }(t 2, s 2) \text { and } s 1=s 2 \\
& \rightarrow \text { incompatiblityInOutputLocationAndShape }(t 1, t 2)
\end{aligned}
$$

More sophisticated incompatibility rules can be found, in particular rules that take into account the possible inferences on spatial relations (e.g. the RCC8 inferences among topological relations).

\section{CONCLUSION}

In this paper we address the general problem of finding usable information visualization techniques in $3 \mathrm{D}$ city models. We propose (1) a model of such techniques and thus a common detailed and organized description of 3D visualization techniques that are too often not explicitly described even when they are widely used, (2) a formalized implementation of this model in the form of an ontology. The ontology and the associated knowledge base can be used to automatically select relevant techniques from the description of the data to display and from a set of specific criteria, taking in account the compatibility or incompatibility between techniques.

The type of ontology that we developed can be evaluated according two ways: the coverage area (data-driven evaluation) and the adequacy for the intended application (applicationbased evaluation) (Brank et al., 2005).

To assess the coverage area we selected a set of visualization techniques described in the scientific literature or widely used in the 3D GIS domain, and created for each of them a representation using the vocabulary defined in the ontology. The experience showed that the defined vocabulary was sufficient to describe those techniques. This is obviously a partial evaluation since there are still other techniques.

Assessing should check the adequacy for the intended application. More precisely it should check that an application searching visualization techniques and detecting inconsistencies is more efficient when using this ontology rather than when using a database, for example. We have limited the evaluation to the effectiveness of the ontology, i.e. to verify that an application that uses this ontology can really (1) Find 3D visualization techniques corresponding to complex research criteria (2) Detect inconsistencies between techniques.

In the near future we will continue to populate the visualization technique knowledge base, with the aim to make it a useful tool for the 3D GIS community.

We also plan to enhance an existing 3D city model visualization tool with implementations of the collected visualization techniques and with a technique selection function that relies on the visualization technique knowledge base.

\section{REFERENCES}

Alamouri, A. and Pecchioli, L., 2010. Retrieving information through navigating in historical Baalbek. In: International 
Archives of the Photogrammetry, Remote Sensing and Spatial Information Sciences, Volume XXXVIII-4/W15, pp. 81-85.

Amar, R.A., Eagan, J., Stasko J.T., 2005. Low-level components of analytic activity in information visualization. In: Proceedings of the 2005 IEEE Symposium on Information Visualization, (InfoVis 2005), p. 111-117.

Amorim, J.H., Valente, J., Pimente1, C., Miranda, A.I. and Borrego, C., 2012. Detailed modelling of the wind comfort in a city avenue at the pedestrian level. In: T. Leduc, G. Moreau \& R. Billen (Eds.), Usage, Usability, and Utility of 3D City Models, 03008, pp. 1-6.

Atila, U., Karas, I. R., Abdul Rahman, A., 2013. Enhancing the visibility of labels in 3D navigation maps. In: Progress and New Trends in 3D Geoinformation Sciences, Lecture Notes in Geoinformation and Cartography (LNG\&C) Series, Pouliot, J.; Daniel, S.; Hubert, F.; Zamyadi, A. (Eds), Springer, Berlin, VI, pp. 249-260.

Bazargan, K., Falquet, G. 2009. Specifying the Representation of Non-geometric Information in 3D Virtual Environments. In Proc. of the 13th international Conference on Human-Computer interaction. (San Diego, CA, July 19-24, 2009), LNCS, J.A. Jacko (Ed.), vol. 5611. Springer-Verlag, Berlin, Heidelberg, pp. 773-782.

Bowman, D. A., Kruijff, E., LaViola, J. J. and Poupyrev, I. 2004. 3D User Interfaces: Theory and Practice. Addison Wesley Longman Publishing Co., Inc., Redwood City, CA, USA.

Brank, J., Grobelnik, M. \& Mladeni, C. D. 2005. A survey of ontology evaluation techniques. In: Proceedings of the Conference on Data Mining and Data Warehouses SiKDD 2005

Brodlie, K., Noor, N. M., 2007. Visualization notations, models and taxonomies. In: I. S. Lim \& D. Duce (Eds.), Theory and Practice of Computer Graphics, Bangor, United Kindom: Eurographics Association, pp. 207-212.

Fisher-Gewirtzman, D., 2012. 3D models as a platform for urban analysis and studies on human perception of space. In: T. Leduc, G. Moreau \& R. Billen (Eds.), Usage, Usability, and Utility of 3D City Models, 01001, pp. 1-16.

Gao, A. S., Nick, J. and Omer, F. R,. 2008. Bringing semantics to visualization services. In: Advances in Engineering Software, Elsevier, vol. 39, Issue 6, June 2008, pp. 514-520.

Gruber, T., 2009. Ontology. In: Encyclopedia of Database Systems, Ling Liu and M. Tamer Özsu (Eds.), Springer-Verlag, Berlin, Heidelberg, pp. 1963-1965, http://tomgruber.org/ writing/ontology-definition-2007.htm (6 May 2013).

Hervy, B., Billen, R., Laroche, F., Carré, C., Servières, M., van Ruymbeke, M., Tourre, V., Delfosse, V. and Kerouanton, J.-L., 2012. A generalized approach for historical mock-up acquisition and data modelling: Towards historically enriched 3D city models. In: T. Leduc, G. Moreau \& R. Billen (Eds.), Usage, Usability, and Utility of 3D City Models, 02009, pp. 1-10.

Hornbæk K, 2006. Current practice in measuring usability: Challenges to usability studies and research. In: Int. J. HumanComputer Studies 64 (2006), pp. 79-102.

Horrocks, I., Patel-Schneider, P. F. \& Boley, H. 2004. SWRL: A Semantic Web Rule Language Combining OWL and RuleML, http://www.w3.org/Submission/2004/SUBM-SWRL20040521/ (28 May 2013).

Lee, B., Plaisant, C., Parr, C. S., Fekete, J.-D., Henry, N., 2006. Task taxonomy for graph visualization. In: Proceedings of the
2006 AVI workshop on BEyond time and errors: novel evaluation methods for information visualization, BELIV '06, New York, NY, USA : ACM, pp. 1-5.

Lu, A., Chen, W., Ribarsky, W. And Ebert, D., 2009. Year-Long Time-Varying 3D Air Quality Data Visualization. In: Advances in Information and Intelligent Sys., Z. W. Ras \& W. Ribarsky (Eds.), Springer-Verlag, Berlin Heidelberg, SCI 251, pp. 289-306.

Marina, O., Leduc, T., Rabino, G. and Karanakov, B., 2012. Integration of urban knowledge through visibilities studies and pedestrian behavior analysis in Old Bazaar in Skopje. In: T. Leduc, G. Moreau \& R. Billen (Eds.), Usage, Usability, and Utility of 3D City Models, 03006, pp. 1-10.

Miller, G. A., 1995. WordNet: A Lexical Database for English. Communications of the ACM, Vol. 38, No. 11, pp. 39-41.

Morell Pérez, A., Pérez Risquet, C., Marx Gómez, J., 2011. An Enhanced Visualization Ontology for a Better Representation of the Visualization Process. In: ICT Innovations 2010, Communications in Computer and Information Science, M. Gusev and P. Mitrevski (Eds.), Springer-Verlag, Berlin Heidelberg 2011, Vol. 83, Part 2, pp. 342-347.

OGC 07-036 (2007) OpenGIS Geography Markup Language (GML) Encoding Standard. C. Portele (ed), Open Geospatial Consortium Inc, http://www.opengeospatial.org/standards/ gml\#downloads (28 May 2013).

OGC 12-019 (2012) OGC City Geography Markup Language (CityGML) Encoding Standard. G. Gröger, T. H. Kolbe, C. Nagel, K.-H. Häfele (eds), Open Geospatial Consortium Inc, http://www.opengis.net/spec/citygml/2.0 (29 May 2013).

OWL Working Group (2012) OWL 2 Web Ontology Language: Document Overview. W3C OWL Working Group (Eds), http://www.w3.org/TR/owl2-overview/ (28 May 2013).

San José, R., Pérez, J.L. and González, R.M., 2012. Advances in 3D visualization of air quality data. In: T. Leduc, G. Moreau \& R. Billen (Eds.), Usage, Usability, and Utility of 3D City Models, 02002, pp. 1-13.

Tyndiuk F., 2005. Référentiels Spatiaux des Tâches d'Interaction et Caractéristiques de l'Utilisateur influençant la Performance en Réalité Virtuelle. Thèse, Université Victor Segalen - Bordeaux II.

Vaaraniemi, M., Freidank, M., Westermann, R., 2013. Enhancing the visibility of labels in 3D navigation maps. In: Progress and New Trends in 3D Geoinformation Sciences, Lecture Notes in Geoinformation and Cartography (LNG\&C) Series, Pouliot, J.; Daniel, S.; Hubert, F.; Zamyadi, A. (Eds), Springer, Berlin, VI, pp. 23-40.

Voigt, M., Pietschmann, S., Grammel, L., Meissner, K., 2012. Context-aware recommendation of visualization components. In: eKNOW 2012, the Fourth International Conference on Information, Process and Knowledge Management, pp. 101109.

Voigt, M., Polowinski, J., 2011. Towards a Unifying Visualization Ontology. Technical report, Institut für Software and Multimediatechnik, Technische Universität Dresden, Germany, March 2011, ISSN: 1430-211X.

Yatskiv, I. and Savrasovs, M., 2012. Microscopic transport model animation visualisation on KML base. In: T. Leduc, G. Moreau \& R. Billen (Eds.), Usage, Usability, and Utility of 3D City Models, 03003, pp. 1-9. 
ISPRS Annals of the Photogrammetry, Remote Sensing and Spatial Information Sciences,

Volume II-2/W1, ISPRS 8th 3DGeoInfo Conference \& WG II/2 Workshop, 27 - 29 November 2013, Istanbul, Turkey

\section{ACKNOWLEDGEMENTS}

The work described in this paper is part of the research "Designing and evaluating 3D knowledge visualization techniques for urban planning" funded by the swiss "Secrétariat d'état à la recherche" (SER No C10.0150) in relation with the COST Action TU0801 "Semantic enrichment of 3D city models for sustainable urban development".

The model used for illustrating some visualization techniques relates to Carouge city (Switzerland) and has been provided by the Service de la mensuration officielle de l'Etat de Genève (SEMO). 